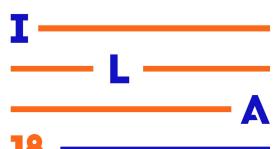

\title{
UTILIZANDO DESIGN PARTICIPATIVO PARA CRIAÇÃO DE MÉTODO DE GERAÇÃO DE SOLUÇÕES TECNOLÓGICAS PARA IDOSOS
}

\section{USING PARTICIPATORY DESIGN FOR CREATION OF GENERATION METHOD OF TECHNOLOGY SOLUTIONS TO ELDERLY}

\author{
Thayssa Maria Silva de Lacerda ${ }^{1}$, Tecnol. Sist. Int. \\ Eduardo Jose Goncalves de Oliveira ${ }^{2}$, Ms. Des.
}

\author{
(1) CESAR School \\ e-mail: thayssa.mlacerda@gmail.com \\ (2) CESAR School \\ e-mail: eduardoloro@gmail.com
}

Design Participativo, Idosos, Método de Criação

Essa pesquisa tem como objetivo relatar a aplicação do Design Participativo em um método de geração de soluções e serviços para idosos. Esse método tem como principal característica o processo de imersão, onde os idosos participam diretamente do processo de Design dos artefatos gerados.

\section{Participatory Design, Elderly, Creation Method}

This research aims to report the application of Participatory Design in a method for generating solutions and services to the elderly. This method has as main characteristic the process of immersion, where the elderly participate directly in the Design process of the generated artifacts.

\section{Introdução}

Segundo o relatório de 2017 da Organização das Nações Unidas, estima-se que o número de pessoas com 60 anos ou mais, cresça de 962 milhões em 2017 para quase 2,1 bilhão em 2050 , principalmente em países em desenvolvimento, representados pela cor laranja no gráfico da Figura 1:

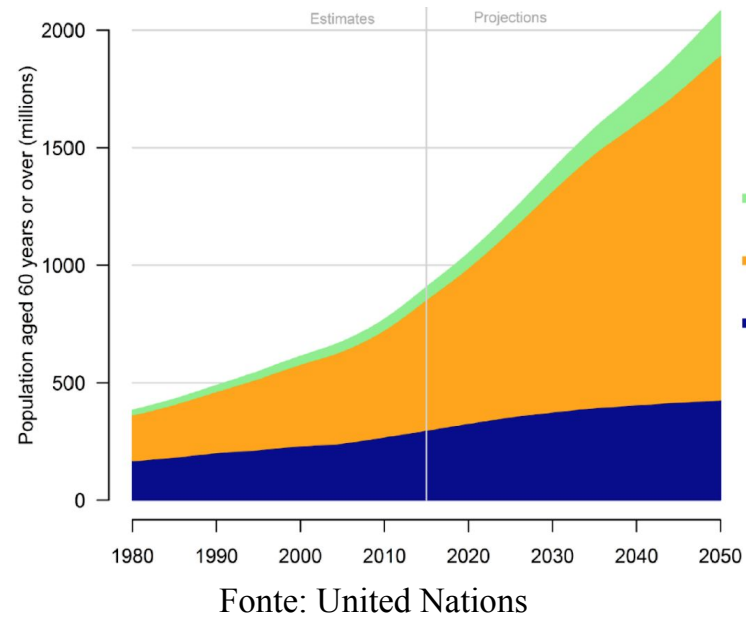

Figura 1. Perspectivas da população mundial de 2017.
Ainda de acordo com levantamento de 2015 da OMS (Organização Mundial de Saúde), esse aumento crescente de cidadãos mais velhos é proporcional à ampliação da necessidade do desenvolvimento de melhores ferramentas e informações que atendam às demandas dos idosos, e mitiguem os efeitos do declínio na saúde e no apoio familiar, além de atender às suas diferentes expectativas.

Devido à evolução constante da tecnologia, existe também a perspectiva de que o uso de artefatos tecnológicos cresça em todos os âmbitos da vida das pessoas, podendo tornar a sociedade cada vez mais dependente desse uso. Esses artefatos possuem a capacidade de auxiliar o público idoso a ser mais independente, melhorar a qualidade de vida, proporcionar o acesso à informação, possibilitar comunicação e pedido de ajuda. Porém, a utilização desses artefatos ainda é mais recorrente no público jovem, com idade entre 18 e 30 anos (MITZNER et al., 2010). Isso ocorre, entre outros fatores, devido aos responsáveis pelo desenvolvimento de muitos 
artefatos digitais continuarem desconsiderando os aspectos fundamentais de acessibilidade e as particularidades desse público (MURIANA, 2017). Esse contexto, além de ser oneroso para quem possui alguma capacidade reduzida, seja por idade ou por outros fatores, vai de encontro ao $4^{\circ}$ Desafio da Sociedade Brasileira de Computação, estabelecido em 2006, que é garantir acesso participativo e universal do cidadão brasileiro ao conhecimento.

Diante disso, fica evidente a importância, durante o desenvolvimento de ferramentas tecnológicas, de considerar os aspectos ergonômicos e de usabilidade, dado os declínios cognitivos, motores e espaciais que os idosos podem apresentar, os quais impactam negativamente na interação com as interfaces dos sistemas computacionais (TAVARES \& SOUZA, 2012). Se devidamente adaptada, a aplicação da metodologia Design Participativo, auxilia na melhora desse cenário, pois viabiliza a identificação e resolução das dificuldades deste público, através de sua participação ativa no processo, possibilitando a criação de soluções a partir de suas experiências pessoais (MURIANA, 2017).

Essa pesquisa tem como objetivo analisar, através de um estudo de caso, como o Design Participativo pode beneficiar a composição de um método de geração de soluções tecnológicas para idosos. Para isso, será abordado o Bulir, um guia criado durante o programa Summer Job, promovido pelo Centro de Estudos e Sistemas Avançados do Recife (CESAR) e patrocinado pela Fundação de Aposentadorias e Pensões dos Servidores do Estado de Pernambuco (FUNAPE), durante a edição 2018.1.

\section{Metodologia da Pesquisa}

Essa pesquisa utiliza como metodologia o Estudo de caso, que segundo Godoy (1995), é uma pesquisa qualitativa que tem como objetivo analisar uma unidade a fundo, através de exame detalhado. Já Yin (1989) define o Estudo de caso como sendo uma forma de pesquisa empírica e investigativa, focada em fenômenos contemporâneos no contexto real de vida e onde são utilizadas múltiplas fontes de evidências.
O estudo de caso tem sido indicado para situações em que a pesquisa pretende atender questionamentos de "como" e "por quê" alguns fenômenos ocorrem, quando se há controle limitado sobre os eventos pesquisados e quando se trata de fenômenos atuais, possíveis de serem analisados somente em contextos reais de vida. No estudo de caso, geralmente é utilizada uma variedade de dados coletados em diferentes momentos, por meio de variadas fontes de informação. Algumas técnicas aplicadas nesta metodologia são pesquisa e observação (GODOY, 1995).

Ainda segundo Godoy (1995), a técnica de observação tem um papel essencial no estudo de caso, pois ela possibilita a apreensão de aparências, eventos e/ou comportamentos. A observação pode acontecer de duas maneiras. A primeira delas é como Participante, onde o observador se coloca na posição dos outros elementos envolvidos no fenômeno em questão. A segunda maneira de observação é como Não-participante, quando o pesquisador atua apenas como espectador atento, registrando todas as ocorrências que interessam ao trabalho.

Dito isso, para descrever como o estudo de caso foi aplicado a esta pesquisa, faz-se necessário definir o fenômeno atual da vida real em questão, que é a criação do método de geração de soluções para idosos, o Guia Bulir. Sobre esse fenômeno, procura-se responder ao questionamento de como os fundamentos do Design Participativo beneficiaram a produção do Guia Bulir, e por quê. Para isso, foram feitas coletas de dados e pesquisa sobre o Design Participativo, além dos dados e relatórios colhidos através de observação de caráter não-participante e acompanhamento, durante todo o processo de desenvolvimento do método.

\section{Metodologia de Design}

Para Bomfim (1995), metodologia é a ciência que se ocupa do estudo de métodos, técnicas e ferramentas, e como se dá suas aplicações na definição, organização e solução de problemas teóricos e práticos, onde, segundo Freitas, Coutinho e Waechter (2013): 


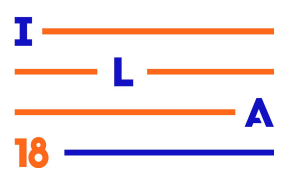

- Método é o caminho pelo qual se atinge um objetivo;

- Técnica consiste em habilidade para execução de determinada ação;

- Ferramentas são instrumentos ou utensílios empregados no cumprimento desta ação.

Bomfim (1995) e Munari (1981) definem a Metodologia de Design como a disciplina que se ocupa da aplicação de métodos a problemas específicos e concretos. Onde métodos e técnicas são instrumentos de ordenação, organização e suporte lógico ao desenvolvimento de um projeto (BOMFIM 1995). Bürdek (2006) defende que teoria e metodologia do Design são reflexos objetivos, tendo seus esforços destinados a otimizar métodos, regras e critérios. Sua utilização dá suporte para a pesquisa, avaliação, validação e otimização no Design. Assim, é possível classificar as Metodologias de Design em metodologias projetuais de Design de Produto, Design Gráfico e Design da Informação (FREITAS, COUTINHO \& WAECHTER 2013).

\subsection{Design Participativo}

A utilização de uma abordagem centrada no usuário, dentro do contexto de desenvolvimento de software e Design de Informação, leva em consideração as características, conhecimentos e experiência dos usuários em potencial, tornando possível uma maior aproximação das expectativas e aceitação desses usuários (LOWDERMILK, 2013). Esse envolvimento pode acontecer em diversos níveis, desde apenas observação, até participação ativa em algumas etapas do processo de Design, de acordo com a possibilidade de atuação (ROGERS, 2013).

Umas das abordagens centradas no usuário é o Design Participativo, que promove um alto grau de envolvimento do usuário, como membro igualitário dentro do processo de Design, de modo que sintam propriedade sobre o que for desenvolvido (MOFFATT, 2004). O Design Participativo surgiu nos anos 70, na Escandinávia, como um desdobramento de uma pesquisa orientada para a ação da época, que estava sendo conduzida com sindicatos locais, para garantir que a introdução da tecnologia não levasse a um desqualificação da força de trabalho. Isso se deu a partir de três projetos iniciais, NJMF, DEMOS, e UTOPIA, onde trabalhadores foram capacitados, recebendo controle ativo do ambiente e processo de trabalho e sendo considerados membros iguais da equipe de Design, participando de todas as etapas do processo (MOFFATT, 2004).

Essa prática (Figura 2) tem como premissa a ideia de que quem é afetado diretamente pelo uso de tecnologia da informação, tem um papel fundamental em sua concepção, gerando conhecimento mútuo por meio de reflexão coletiva. Isso se dá através do envolvimento das pessoas interessadas nas tarefas que fazem parte das etapas do processo de Design, além dos próprios designers (BJERKNES \& BRATTETEIG, 1995).

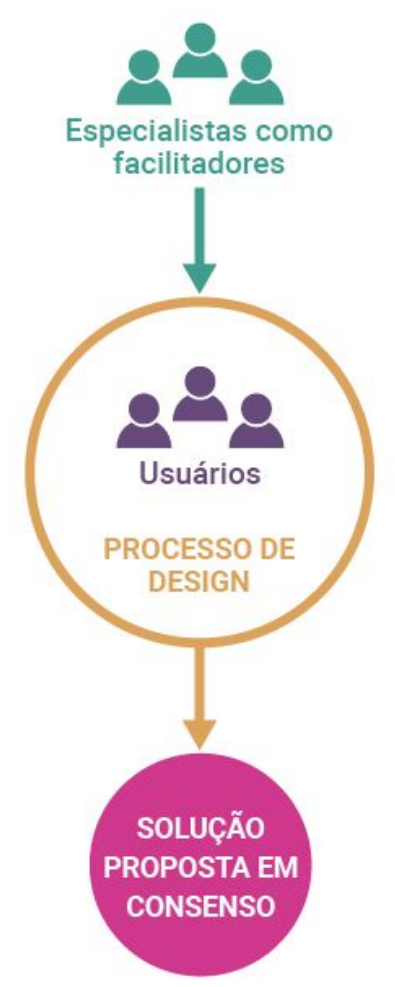

Fonte: Autores da pesquisa

Figura 2. Prática do Design Participativo.

Dentro do contexto de Design Participativo aplicado com idosos, Anna Dickinson e Dewsbury Guy (2006) afirmam que utilizar as suas vastas experiências, ao tentar atender às suas necessidades, é uma abordagem mais propensa a ser bem-sucedida para o desenvolvimento de um novo produto ou serviço, ao invés de confiar apenas em padrões de 


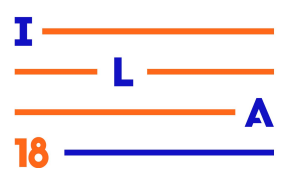

interação baseados em paradigma do computador. Mas, para isso, é preciso uma análise prévia da experiência individual de cada pessoa que irá participar do processo, visto que essas experiências influenciam nos resultados e como essas pessoas vão contribuir. Por isso, incluir diferente usuários não é uma tarefa trivial (MURIANA, 2017). Os papéis e as relações com as tarefas de Design variam, assim como as necessidades, de acordo com as características do participante, como o estágio da vida, condições físicas e cognitivas, e por outros atributos (MULLER, DRUIN, 2012).

Além disso, Halskov e Hansen (2014) apresentam os aspectos fundamentais do Design Participativo, que serviram como critério de avaliação dos aspectos encontrados no Guia Bulir. Aspectos esses que são:

- Político: as pessoas que são afetadas por uma decisão deveriam ter a oportunidade de influenciá-la;

- Pessoas: as pessoas possuem papel crítico no processo de Design por serem experts em suas próprias vidas;

- Contexto: a situação de uso é um ponto inicial fundamental para o processo de Design;

- Métodos: os métodos são meios para os participantes influenciarem o processo de Design;

- Produto: o objetivo da participação é realizar Designs alternativos para melhorar a qualidade de vida.

\section{O Guia Bulir}

O Bulir foi nomeado a partir de um termo provindo da linguagem nordestina, sendo definido pelo Dicionário Aurélio (2010) como o ato de mexer nas coisas, conhecer, mover e agitar. Trata-se de uma ferramenta que visa proporcionar o empoderamento do idoso, através do estímulo à geração de ideias para solução de problemas reais de pessoas nessa faixa etária, e foi desenvolvido durante o Summer $J o b$, programa promovido pelo CESAR, que busca propiciar experiência prática para estudantes de graduação, através do desenvolvimento de soluções para desafios reais propostos por patrocinadores. Esse programa acontece em duas edições a cada ano, no período de férias dos estudantes. O processo operacional de desenvolvimento proposto pelo Yearbook Summer Job (2018) (Figura 3) tem duração de 6 semanas, divididos nas seguintes etapas:

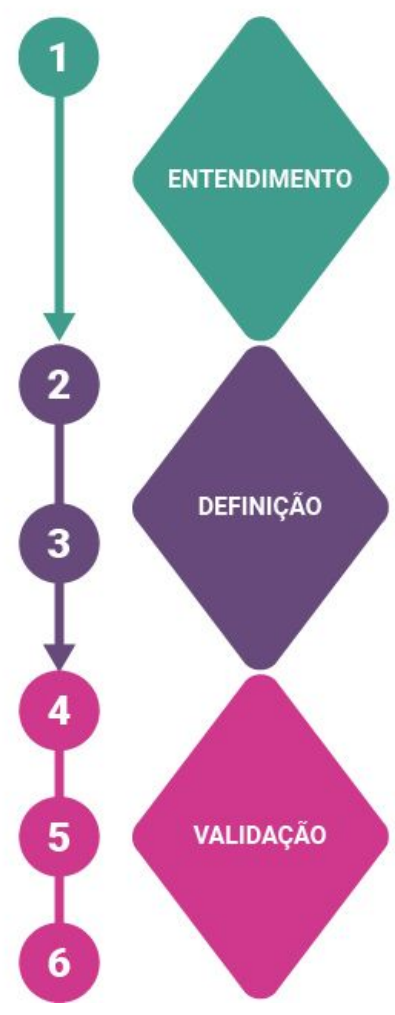

Fonte: Autores da pesquisa

Figura 3. Processo de desenvolvimento do Programa

CESAR Summer Job.

Onde, a primeira semana é voltada para o entendimento, aprofundamento e validação do problema e/ou oportunidade (pesquisas e estudo de campo). Em seguida, nas duas semanas seguintes, são definidas as soluções para o problema trabalhado, considerando o prazo, o usuário/consumidor, a complexidade de desenvolvimento e a viabilidade. Por fim, nas três últimas semanas, as soluções selecionadas são prototipadas e validadas.

Em meio a esse processo, são feitas entregas parciais semanais, com exceção da quinta semana, totalizando quatro entregas parciais e uma final. Ao 
Interaction Latin America

Rio de Janeiro, Brasil - 2018

http://ila.ixda.org/2018

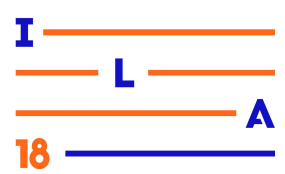

recomendado pelo método que, seja feito o recrutamento dos participantes que se encaixem com os perfis identificados no processo de pesquisa.

final, é feita a apresentação das soluções para os patrocinadores e demais grupos em formato de Pitch, que segundo Martin Soorjoo (2012), trata-se de uma apresentação direta, onde o objetivo de convencer um possível investidor da relevância e perspectivas positivas em termos de negócio da solução proposta. Nele, é importante ressaltar os aspectos mais importantes da proposta. Entre esses pontos, o problema a ser resolvido, a situação do mercado em relação ao problema, qual o diferencial da solução proposta e os profissionais envolvido no desenvolvimento.

Dentre os 12 patrocinadores, a FUNAPE trouxe como desafio a inserção digital para idosos, através da identificação de serviços relevantes para esse público. A partir do desafio apresentado, a equipe responsável, através de imersão e estudos sobre o tema, desenvolveu o Guia Bulir, um método que funciona como uma ferramenta de geração de ideias de produtos e serviços tecnológicos para idosos, e tem como principal característica a inserção de pessoas da terceira idade, principais atingidas pelas ideias a serem geradas, no processo de criação. A partir do uso do guia, idosos ligados à FUNAPE se tornam os principais agentes geradores de soluções para seus desafios diários, a partir das suas experências pessoais. Através disso, o Guia Bulir propõe proporcionar empoderamento aos idosos, pois eles passam a ter propriedade sobre o que for criado, além de acarretar em resultados mais eficazes e coerentes, considerando as particularidades desse público.

O Guia Bulir é constituído pelas diretrizes básicas necessárias para a execução do método, onde são listados: os materiais necessários, os perfis e papéis de cada participante e as orientações para o facilitador, um funcionário da própria FUNAPE, que irá conduzir todo o processo. Além disso, o guia é dividido em 4 etapas de execução:

- Mobilização, onde são escolhidos o tema a ser abordado nas etapas seguintes. Segundo o Guia, o tema não possui delimitação de área de atuação, deve ser objetivo, com nível de complexidade compatível com os perfis dos participantes e apresentar relação direta com o cotidiano e contexto dos idosos participantes. Posteriormente, é
- Realização, onde ocorre a participação ativa e direta dos idosos recrutados e a geração de ideias, através da identificação das demandas, montagem de contexto e cenário, realização de contação de histórias, filtragem das histórias contadas, geração da jornada do usuário, proposta de soluções, execução de Brain Draw, onde cada participante dispõe de uma folha A4 e 10 minutos para desenhar uma solução para a dor encontrada durante a etapa de jornada do usuário. Feito isso, deverão colar estas soluções em uma parede para que todos visualizem de forma mais clara possível. Nesta etapa, cada um pode levantar a apresentar as suas soluções. Além disso, essa etapa contempla a convergência das propostas similares, a seleção das melhores alternativas e montagem da estrutura da apresentação.

- Apresentação, onde são apresentadas as soluções geradas pelos participantes.

- Seleção, onde os organizadores analisam as soluções, baseada nos critérios impacto $\mathrm{x}$ recursos. Os idosos não participam dessa etapa.

Ainda de acordo com o Guia Bulir, após a finalização das etapas de geração de ideias, as soluções apresentadas são analisadas, através de matriz de análise (Figura 4), que permite identificar qual é mais representativa dentro do contexto estabelecido. 


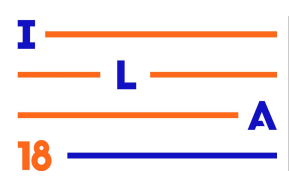

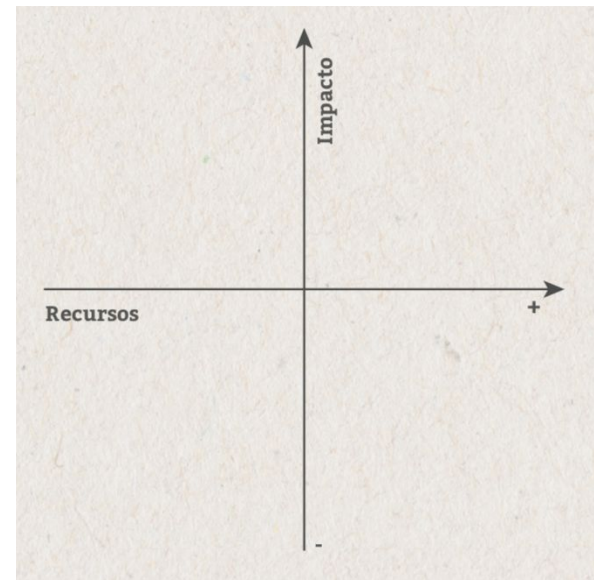

Fonte: Guia Bulir

Figura 4. Matriz de análise de soluções.

As soluções mais representativas são aquelas posicionadas no quadrante superior esquerdo, ou seja, quelas que necessitam de menos recursos e, ao mesmo tempo, causam mais impacto. Essa é considerada uma etapa interna do processo, pois os idosos participantes das etapas anteriores não são envolvidos, apenas colaboradores e agentes estratégicos da FUNAPE.

O Guia propõe que as soluções escolhidas devem ser testadas e aperfeiçoadas, levando em consideração o seu público-alvo, os idosos, tornando possível impactos reais na vida deles.

\subsection{Design Participativo no Bulir}

As abordagens iniciais, adotadas durante a primeira semana do programa, foram voltadas a pesquisas, com objetivo de aprofundamento e entendimento do desafio proposto pela FUNAPE, que possui 64.004 aposentados vinculados às suas atividades: Inserção Digital para Idosos e Identificação de serviços relevantes.

Nessa etapa foi realizado o focus group, com o objetivo de expandir o entendimento sobre o público-alvo da pesquisa. Segundo Thomas Greenbaum (1998), trata-se de uma técnica de pesquisa qualitativa, cuja sessões são conduzidas por um mediador treinado, que mantém a conversa próxima ao tópico proposto e faz perguntas relevantes. Além disso, deve ser composto por um grupo de pessoas razoavelmente homogêneo, com características que casam com os objetivos e critérios da pesquisa.

O focus group foi realizado com 16 idosos que fazem parte do programa Vida Ativa, dentre eles $25 \%$ homens e $75 \%$ mulheres. Segundo dito em entrevista ao blog de notícias do Governo do Estado de Pernambuco (2017), pela presidente da FUNAPE, Tatiana Nóbrega, "o Programa Vida Ativa busca valorizar e integrar os aposentados e pensionistas da previdência do Estado, proporcionando-lhes oportunidade de convivência e aprendizado, através de atividades educacionais, de cultura e lazer, visando uma melhor qualidade de vida". Através da sessão promovida pelos autores do Guia, foi possível identificar os perfis de idosos de acordo com sua familiaridade com a tecnologia e artefatos tecnológicos, bem como em relação aos interesses pessoais no aprendizado e utilização.

Como é proposto na Figura 5, há uma dinâmica entre os perfis, onde quem possui características mais propensas ao uso e aprendizado proativo de novas tecnologias e costuma utilizar serviços e produtos digitais, chamado de "Antenado", era capaz de influenciar os participantes classificados como "Curiosos" a aprenderem e utilizarem mais tais ferramentas. Os "Curiosos", apesar de apresentarem disposição para aprender, não tinham ativismo no uso desses artefatos quanto os "Antenados". O terceiro perfil identificado como "Haters", tem como principal característica a falta de interesse em utilizar e aprender sobre artefatos tecnológico, porém acabaria também sendo influenciado pela dinâmica, através da troca de experiências com os outros perfis. 


\section{entrada mediada pelo uso de plataforma tecnológica}

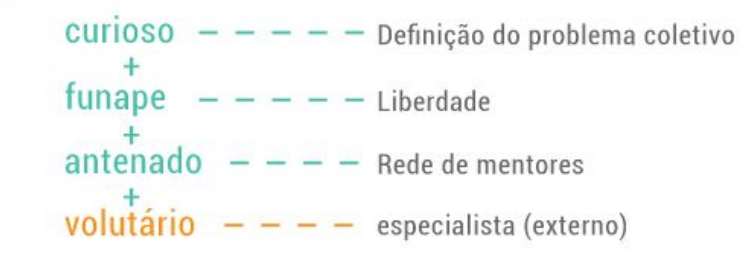

saída mediada pelo uso de plataforma tecnológica

$=$ Gerar empoderamento digital para os idosos



Fonte: Adaptação a partir do Guia Bulir

Figura 5. Perfis de idosos e dinâmica de aprendizado.

Este processo de identificação de perfis, onde aspectos e experiências pessoais de cada participante foram levantados e entendidos, sob o critério do desafio proposto, foi o primeiro elemento viabilizador do Design Participativo no método Bulir, visto que, como afirmam Muller, Durin (2012) e Muriana (2017), essas experiências influenciam nos resultados e em como essas pessoas vão contribuir com a metodologia. Por isso, a seleção das pessoas não é trivial e deve ser feita a partir de uma análise prévia. Através dessa análise, foi possível selecionar os perfis adequados para as sessões do Bulir, os "Antenados" e "Curiosos", atendendo a dois aspectos fundamentais do Design Participativo apresentados por Halskov e Hansen (2014): o aspecto Político, que afirma que as pessoas que são afetadas por uma decisão deveriam ter a oportunidade de influenciá-la; e o aspecto Pessoas, que afirma que as pessoas possuem papel crítico no processo de Design por serem experts em suas próprias vidas.

Quando analisado sob o aspecto fundamental do Design Participativo chamado Contexto, que afirma que a situação de uso é um ponto inicial fundamental para o processo de design (HALSKOV \& HANSEN, 2014), e sob o critério de envolvimento dos usuários, de maneira ativa e igualitária, apontado por Moffatt (2004), Bjerknes e Bratteteig (1995), e Muriana (2017), as etapas de
Realização e Apresentação propostas pelo Guia Bulir também apresentam conceitos de Design Participativo, visto que as técnicas propostas são de responsabilidade dos idosos recrutados para as rodadas.

Dentre essas responsabilidades estão contar uma história de seu cotidiano que seja relacionada ao tema apresentado pelo facilitador da FUNAPE, a partir dessas histórias, montar a jornada do usuário, escolher qual problema será tratado dentro das dores levantadas, desenhar soluções para esse problema e selecionar a melhor alternativa dentre as propostas, preparar uma apresentação, respondendo os questionamentos: Quem é a pessoa que irá desfrutar da sua ideia? Como funciona a ideia? Funcionará de que forma? Em que momento sua ideia será usada? e por fim, um membro do grupo deve apresentar a solução co-criada.

Borges et al. (2012) afirmam que a abordagem de design participativo viabiliza aos usuários uma imersão em seu contexto de vida, trazer das suas experiências e idealizações, dentro do que se refere às suas necessidades sobre as soluções em questão. Essa viabilização pode identificada dentro da descrição das responsabilidades propostas pelo método Bulir, visto que as soluções são desenvolvidas a partir de histórias do cotidiano dos participantes, que têm relação com o tema proposto. Por sua vez, como proposto pelos autores do Guia Bulir, a escolha do tema deve apresentar relação direta com o cotidiano e contexto dos idosos participantes.

Já do ponto de vista do aspecto fundamental Métodos de Design Participativo, que afirma que os métodos são meios para os participantes influenciarem o processo de design (HALSKOV \& HANSEN, 2014), visto que o Design Participativo é uma abordagem centrada no usuário (MOFFATT, 2004), é possível identificar nas ferramentas propostas para a etapa de execução a viabilização da influência dos idosos. Na etapa de execução são propostas as seguintes técnicas:

- Storytelling: é uma técnica de compartilhamento de histórias, onde os participantes podem comentar histórias de outros participantes e podem enumerar 


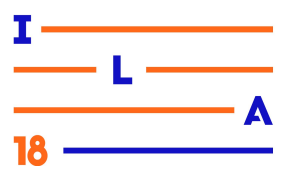

pontos em comum ou incomuns (MULLER et al. 1997). Para Muller \& Druin (2012), as histórias em atividades participativas podem ter 4 funções. A funções encontradas no Método Bulir são: Primeiramente, servirem como tópicos para iniciar uma conversa, análises ou dar feedback. A segunda função é ser utilizada pelos usuários finais como parte de suas contribuições para o conhecimento requisitado para entender a oportunidade de um produto ou serviço e para especificar o que produtos ou serviços deveriam fazer. As histórias compartilhadas podem ainda serem utilizadas por times heterogêneos para apresentar seus conceitos do que o design de um serviço ou produtos irá fazer, como ele será usado e quais alterações irão ocorrer como um resultado.

- BrainDraw: consiste em cada participante iniciar o desenho relacionado ao conceito da solução que se deseja desenvolver. Ao final de um tempo pré-determinado, cada participante move seu desenho para o participante que está ao seu lado ou se desloca até o próximo desenho e continua realizando o design que recebeu do outro participante. O processo é realizado até que os participantes estejam satisfeitos (DRAY, 1992). Dentro do Guia Bulir, a técnica foi adaptada para que as soluções desenhadas sejam apresentadas em uma parede, onde todos visualizem e identifiquem o que suas soluções têm em comum. A partir disso, o facilitador incentiva a fusão de soluções para que se chegue a uma decisão coletiva.

- Jornada do usuário: É uma representação gráfica das etapas de relacionamento do cliente (Figura 6), chamados pontos de contato, com um produto ou serviço, que vai descrevendo os passos chave percorridos antes, durante e depois da compra e utilização. Essa técnica pode ser utilizada para entender o ciclo de relacionamento do cliente com um produto ou serviço. Ao mapear essas etapas é possível analisar suas expectativas em cada momento, viabilizando a criação de formas de atendê-las melhor (VIANNA, 2012).

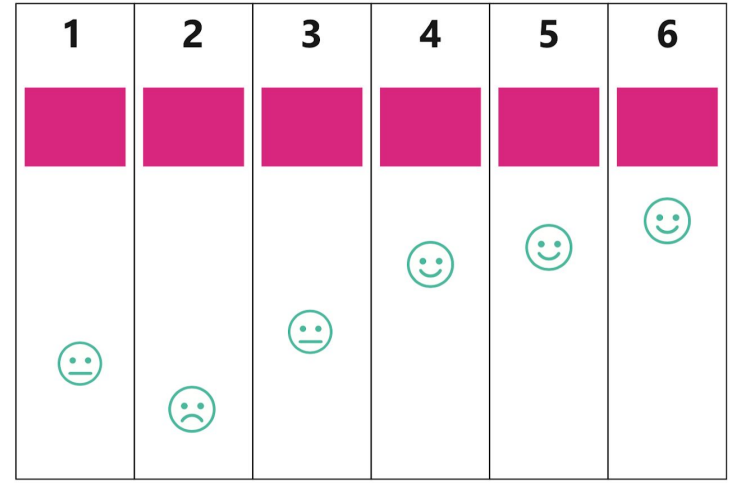

Fonte: Autores do Guia Bulir

Figura 6. Mapa da Jornada do Usuário

\subsection{Resultados Gerados}

O objetivo do Design Participativo, descrito no aspecto fundamental Produto, é desenvolver Designs alternativos para melhorar a qualidade de vida dos participantes (HALSKOV \& HANSEN, 2014). A dinâmica do Guia Bulir, por ser um instrumento de auxílio na solução dos problemas dos idosos, através das suas próprias vivências e inserção ativa no processo de geração de ideias, também compartilha do mesmo objetivo, visto que, como afirma Mitzner et al. (2010), o uso de serviços e artefatos tecnológicos promove a qualidade de vida dos idosos.

Outro fator de melhora de qualidade de vida observado, como o Guia Bulir aponta, é que partir dos estudos, entrevistas e focus group, foi possível identificar que os idosos entrevistados se sentem orgulhosos por se sentirem responsáveis por administrar grupos ou cuidar de alguma demanda pessoal, por isso, o Bulir, pode ser um meio de proporcionar esse sentimento, umas vez que os mesmos poderão pensar, adaptar e estudar suas ideias e soluções.

Como o processo de geração de ideias, proposto pelo Guia Bulir (Figura 7) oferece a participação estruturada, facilitada e interpretada em direção a futuras práticas de design, é possível afirmar que, a participação dos idosos também pode auxiliar na usabilidade das soluções geradas (BODKER, GRONBAEK \& KYNG 1993). Onde, entende-se usabilidade como sendo um atributo de qualidade que avalia a facilidade de utilização das interfaces 


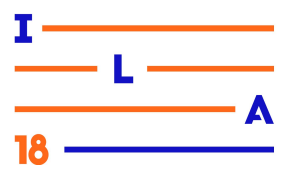

do usuário, ou refere-se a métodos facilitadores de uso, durante o processo de design (NIELSEN, 2003).

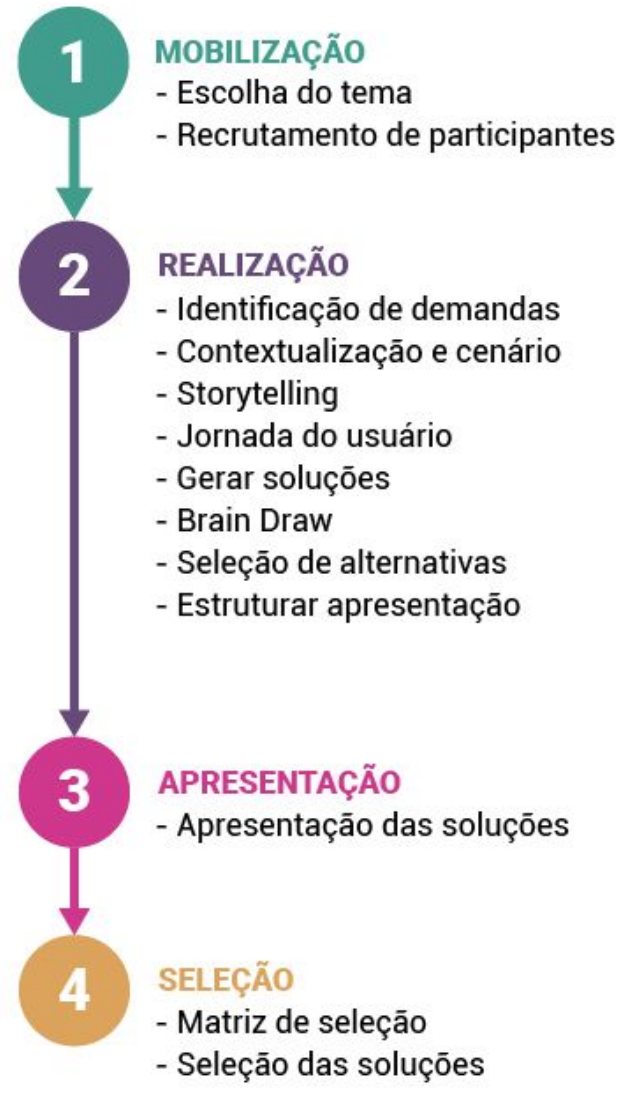

Fonte: Adaptação a partir do Guia Bulir Figura 7. Processo do Guia Bulir

Segundo Nielsen (2003), existem 5 componentes de qualidade de usabilidade:

- Aprendizagem: facilidade de uso e realização de tarefas pelos usuários, para no primeiro contato com a interface;

- Eficiência: quando os usuários aprendem o design com a mesma rapidez que eles executam tarefas;

- Memorabilidade: Facilidade em restabelecer a proficiência, quando os usuários retornam a interface após um período sem usá-lo;

- Erros: quando o número de ocorrências de erros que o usuários cometem é pequena, a severidade desses erros é baixa;
- Satisfação: Quando o Design é agradável para o usuário.

Somado a isto, o Guia Bulir tem como principal proposta a viabilização do protagonismo no processo de construção, causando maior engajamento, e com isso, os resultados tendem a serem mais eficazes, aumentando a aceitação e, consequentemente, a utilização das soluções geradas pelos idosos (TEIXEIRA, 2018), corroborando com a filosofia do Design Participativo levantada por Moffatt (2004).

\section{Conclusão}

Os dados e fenômenos observados, a partir da participação como mentora de Design da equipe, durante o processo de desenvolvimento do Guia Bulir, e através dos estudos levantados nessa pesquisa, sugerem que o método, por ser constituído de princípios fundamentais do Design Participativo, pode ser um agente motivador de engajamento $\mathrm{e}$ interesse para os idosos participantes do Vida Ativa que testaram o método.

Porém, como não foram desenvolvidas soluções criadas a partir da utilização do Guia até o momento dessa pesquisa, um estudo da relevância e eficiência, sob a ótica de usabilidade e interação, dos artefatos gerados é sugerido como trabalho futuro. Outro ponto passível de mais estudos seria a aplicação dos fundamentos do Design Participativo em outras etapas de um processo de desenvolvimento, e não somente na fase de geração de ideias e entendimento do contexto dos usuários, dores e problemas enfrentados.

\section{Referências Bibliográficas}

BJERKNES, Gro; BRATTETEIG, Tone. User participation and democracy: A discussion of Scandinavian research on system development. Scandinavian Journal of information systems, v. 7, n. 1, p. 1, 1995.

BODKER, S.; GRONBAEK, K.; KYNG, M. Cooperative design: techniques and experiences from the Scandinavian scene. In: SCHULER, D.; NAMIOKA, A. (Ed.). Participatory design: principles and practices. Hillsdale: Erlbaum, 1993. 


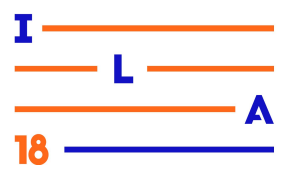

BOMFIM, Gustavo A. Metodologia para desenvolvimento de projetos. Editora Universitária/UFPB, 1995.

BORGES, Luciana Correia Lima de Faria et al. Customizing a communication device for a child with cerebral palsy using participatory design practices: contributions towards the PD4CAT method. Proceedings of the 11th Brazilian Symposium on Human Factors in Computing Systems. Brazilian Computer Society. p. 57-66, 2012.

BOSCARIOLI, C.; ARAÚJO, R. M.; MACIEL, R. S. P. I GranDSI-BR - Grand Research Challenges in Information Systems in Brazil 2016-2026. Special Committee on Information Systems (CE-SI). Brazilian Computer Society (SBC), 2017.

BÜRDEK, Bernhard E. Design-história, teoria e prática do design de produtos. Editora Blucher, 2010.

CESAR. Summer Job Yearbook 2018.1. Centro de Estudos e Sistemas Avançados do Recife, 2018. Disponível em: https://goo.gl/E52nVe Acessado em: março, 2018.

DE FREITAS, R. F.; COUTINHO, S. G.; DA WAECHTER, H. N. Análise de Metodologias em Design: a informação tratada por diferentes olhares. Estudos em Design, v. 21, n. 1, 2013.

DICKINSON, Anna; DEWSBURY, Guy. Designing computer technologies with older people. Gerontechnology, 2006.

DRAY, S. M. Understanding and supporting successful group work in software design: Lessons from IDS [Position paper]. I. Karat and I. Bennett,(Chairs), Understanding and supporting successful group work in software design.

Workshop at CSCW'92 conference, Toronto, 1992.

GODOY, Arilda Schmidt. Pesquisa qualitativa: tipos fundamentais. Revista de Administração de empresas, v. 35, n. 3, p. 20-29, 1995.

GOVERNO DO ESTADO DE PERNAMBUCO. Beneficiários do Programa Vida Ativa da
FUNAPE têm aula de inglês na exposição beatlemania experience. Blog de Notícias, 2017. Disponível em: http://www.pe.gov.br/b/15613 Acessado em: maio, 2018.

GREENBAUM, Thomas L. The handbook for focus group research. Sage, 1998.

HALSKOV, Kim; HANSEN, Nicolai Brodersen. The diversity of participatory design research practice at PDC 2002-2012. International Journal of Human-Computer Studies, v. 74, p. 81-92, 2015.

HOLANDA, Aurélio Buarque de et al. Dicionário Aurélio. Salvador: Positivo Editora, 2010.

LOWDERMILK, Travis. User-centered design: a developer's guide to building user-friendly applications. " O'Reilly Media, Inc.", 2013.

MITZNER, Tracy L. et al. Older adults talk technology: Technology usage and attitudes. Computers in human behavior, v. 26, n. 6, p. 1710-1721, 2010.

MOFFATT, Karyn. Designing technology for and with special populations: and exploration of participatory design with people with aphasia. Tese de Doutorado. University of British Columbia, 2004.

MULLER, M. L.; DRUIN, A. Participatory Design: The Third Space in HCI. In Jacko, J. A. (ed.) Human-Computer Interaction Handbook, Third Edition, CRC Press, p. 1125-

1154, 2012.

MULLER, Michael J.; HASLWANTER, Jean Hallewell; DAYTON, Tom. Participatory practices in the software lifecycle. Handbook of Human-Computer Interaction (Second Edition), p. 255-297, 1997.

MUNARI, Bruno. Das coisas nascem coisas, 1981.

MURIANA, Luã Marcelo et al. Incluindo idosos com comprometimento cognitivo no design participativo: avaliação e adaptações de técnicas de design, desafios e lições aprendidas. 


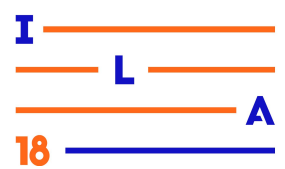

Dissertação de Mestrado. Universidade Estadual de Campinas. 2017.

NIELSEN, Jakob. Usability 101: Introduction to usability. 2003. Disponível em: https://www.nngroup.com/articles/usability-101-intr oduction-to-usability/ Acessado em: junho, 2018.

ROGERS, Yvonne; SHARP, Helen; PREECE, Jennifer. Design de Interação. Bookman Editora, 2013.

SOORJOO, Martin. Here's the Pitch: How to Pitch Your Business to Anyone, Get Funded, and Win Clients. John Wiley \& Sons, 2012.

TAVARES, M. M. K.; SOUZA, S. T. C. Os idosos $e$ as barreiras de acesso às novas tecnologias da informação e comunicação. RENOTE, 10(1), 2012.

TEIXEIRA, Marcolina. Gerações unidas pela era digital. Jornal Diário de Pernambuco, 2018.

Disponível em:

http://www.impresso.diariodepernambuco.com.br/a pp/noticia/cadernos/vida-urbana/2018/02/22/interna vidaurbana, $183832 /$ geracoes-unidas-pela-era-digita 1.shtml Acessado em: abril, 2018.

UNITED NATIONS. World population ageing 2017. Department of Economic and Social Affairs Population Division, 2017. Disponível em:

http://www.un.org/en/development/desa/population/ publications/pdf/ageing/WPA2017 Report.pdf

Acessado em: maio, 2018.

VIANNA, Maurício. Design thinking: inovação em negócios. Design Thinking, 2012.

VIN, R. K. Case study research: design and methods. Newbury Park, CA: Sage Publications, p. 23, 1989.

WORLD HEALTH ORGANIZATION. World Report on Ageing and Health. World Health Organization, 2015.

\section{Agradecimentos}

À Ana Caroline Nascimento, Jessica Lima, Laíza Amorim e Victor Miranda de Melo, participantes do Summer Job 2018.1 e criadores do Guia Bulir.

Ao CESAR, provedor do material utilizado nessa pesquisa.

À FUNAPE, patrocinadora do projeto estudado nesta pesquisa. 